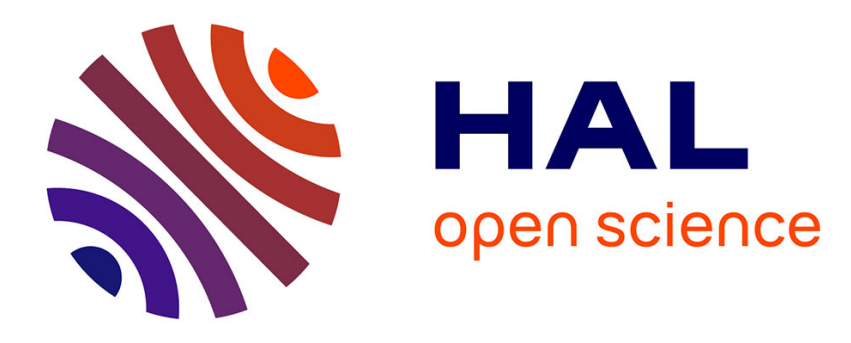

\title{
Les certifications en Espagnol Langue Étrangère sur le marché français
}

Carlos Meléndez Quero

\section{To cite this version:}

Carlos Meléndez Quero. Les certifications en Espagnol Langue Étrangère sur le marché français. Recherche et Pratiques Pédagogiques en Langues de Spécialité: Cahiers de l'APLIUT, 2012, XXXI (3), pp.42-62. 10.4000/apliut.3042 . hal-00752673

\section{HAL Id: hal-00752673 https://hal.science/hal-00752673}

Submitted on 7 Jan 2020

HAL is a multi-disciplinary open access archive for the deposit and dissemination of scientific research documents, whether they are published or not. The documents may come from teaching and research institutions in France or abroad, or from public or private research centers.
L'archive ouverte pluridisciplinaire HAL, est destinée au dépôt et à la diffusion de documents scientifiques de niveau recherche, publiés ou non, émanant des établissements d'enseignement et de recherche français ou étrangers, des laboratoires publics ou privés. 
Cahiers de l'Apliut

Vol. XXXI N 3 | 2012

La certification en langues - État des lieux

\section{Les certifications en Espagnol Langue Étrangère sur le marché français}

Las certificaciones de Español como Lengua Extranjera en el mercado francés

\section{Carlos Meléndez Quero}

\section{(2) OpenEdition}

Journals

\section{Édition électronique}

URL : http://journals.openedition.org/apliut/3042

DOI : 10.4000/apliut.3042

ISSN : 2119-5242

Éditeur

APLIUT

\section{Édition imprimée}

Date de publication : 15 octobre 2012

Pagination : 42-62

ISSN : 2257-5405

Ce document vous est offert par Université de Lorraine

\section{UNIVERSITÉ}

DE LORRAINE

\section{Référence électronique}

Carlos Meléndez Quero, "Les certifications en Espagnol Langue Étrangère sur le marché français », Recherche et pratiques pédagogiques en langues de spécialité [En ligne], Vol. XXXI №3 | 2012, mis en ligne le 25 octobre 2012, consulté le 07 janvier 2020. URL : http://journals.openedition.org/apliut/3042 ; DOI : 10.4000/apliut.3042 


\section{Carlos Meléndez Quero}

\section{Université de Lorraine}

\section{Les certifications en Espagnol Lan-} gue Étrangère sur le marché français

\author{
Mots-clés \\ certifications, CLES, DCL, BULATS, \\ DELE, évaluation, critère, compétence en \\ langue, instructions ministérielles, ensei- \\ gnement, apprentissage, espagnol langue \\ étrangère
}

\section{Résumé}

Dans un contexte difficile, marqué par les contradictions des discours officiels exigeant des lauréats aux concours de recrutement des personnels enseignants des premier et second degrés l'obtention d'un certificat de langue étrangère de niveau B2 du Cadre européen commun de référence pour les langues, cet article présente une comparaison des quatre certifications acceptées actuellement par le Ministère de l'Éducation Nationale pour attester de la compétence en langue espagnole (CLES, DCL, BULATS et DELE). Dans ce but, suivant les critères d'évaluation de l'utilité d'un test de Bachman et Palmer (1996) et fondé sur notre expérience professionnelle dans le domaine de l'évaluation des certifications d'espagnol langue étrangère, ce travail analyse plusieurs critères (fiabilité, validité, authenticité, interactivité, impact et faisabilité) pouvant orienter le choix des enseignants et des candidats d'espagnol quant au certificat, diplôme ou test de langue à adopter.

\section{Las certificaciones de Español como Lengua Extranjera en el mercado francés}

\section{Palabras clave}

certificaciones, CLES, DCL, BULATS, DELE, evaluación, criterios, competencia en lengua, instrucciones ministeriales, enseñanza, aprendizaje, español como lengua extranjera

\section{Resumen}

En un contexto dificil, marcado por las contradicciones de los discursos oficiales que exigen a las personas que aprueben las oposiciones para personal docente de infantil, primaria y secundaria la obtención de un certificado de lengua extranjera de nivel B2 del Marco común europeo de referencia para las lenguas, este artículo presenta una comparación de las certificaciones aceptadas por el Ministerio de Educación Nacional para justificar la competencia en lengua española (CLES, DCL, BULATS y DELE). Con este fin, siguiendo los criterios de evaluación de la utilidad de un test de Bachman y Palmer (1996) y basado en nuestra experiencia profesional en el dominio de la evaluación de las certificaciones de español como lengua extranjera, este trabajo analiza varios criterios (fiabilidad, validez, autenticidad, interactividad, impacto y factibilidad) que pueden orientar a los profesores y candidatos de español en la elección de un certificado, diploma o test de lengua. 


\section{Les certifications en Espagnol Langue Étrangère sur le marché français}

\section{Introduction}

Présenter la situation des certifications des langues étrangères en France ne constitue pas une tâche facile, en raison des changements dans les dispositions administratives auxquels les enseignants et les apprenants ont été confrontés dernièrement ; il convient de garder présentes à l'esprit les interrogations que suscitent les évolutions des consignes officielles et de rappeler l'inquiétude des étudiants qui préparent les concours nationaux de recrutement de personnels enseignants, ainsi que la confusion des professeurs de langues vivantes par rapport aux incohérences des discours tenus ${ }^{1}$. Une fois ce contexte difficile exposé, nous partirons des critères d'évaluation de Bachman et Palmer (1996) pour tenter de comparer les certificats, diplômes et tests acceptés pour attester du niveau B2 de maîtrise en langue espagnole : CLES (Certificat de compétences en langues de l'enseignement supérieur), DCL (Diplôme de compétence en langue), BULATS (Business Language Testing Service) et DELE (Diploma de Español como Lengua Extranjera).

\section{Les instructions officielles sur les certifications en langues étrangères}

L'arrêté du 31 mai 2010 (JORF n 0139 du 18 juin 2010) exige des futurs enseignants n'ayant pas suivi une formation dans le domaine des langues étrangères l'obtention du « certificat de compétences en langues de l'enseignement supérieur de deuxième degré » ou de «toute autre certification [...] attestant de la maîtrise d'une langue étrangère à un niveau de qualification correspondant au moins au niveau B2 du cadre européen commun de référence pour les langues ».

La parution de ce texte a entraîné une préoccupation visant à intégrer les langues vivantes aux formations préparant aux métiers de l'enseignement et à orienter les cours vers la préparation aux certifications ${ }^{2}$. Or, malgré la publication d'une liste de certifications équivalentes au CLES 2, le début de l'année universitaire 2011-

\footnotetext{
${ }^{1}$ Toutes les informations contenues dans cette contribution concernent la situation des langues vivantes en France à la date de révision de l'article (septembre 2012).

2 «Sauf à trouver une forme de test à laquelle on ne puisse entraîner les candidats, force est de reconnaître que la forme de l'évaluation finale modélise bel et bien les pratiques en amont 》 (Association pour la Recherche en Didactique de l'Anglais et en Acquisition, en ligne: $<$ http://ardaarda.canalblog.com/archives/2011/11/index.html $>$ ).
} 
2012 en France a été marqué par l'angoisse des candidats aux concours, dont le niveau en langue étrangère est, pour beaucoup, très inférieur à celui exigé par les instructions officielles.

Cependant, le site ministériel semble remettre en question l'obligation de produire la certification en langue étrangère pour les futurs enseignants. Outre qu'elle dispense de cette obligation les étudiants ayant suivi des programmes Erasmus, la dernière mise à jour $\mathrm{du}$ site ministériel en septembre 2012 $<$ http://www.education.gouv.fr/cid56907/certificats-exiges-a-la-titularisation-deslaureats-des-concours-de-professeurs-des-ecoles.html $>$ indique que des crédits acquis dans des unités d'enseignement de langues vivantes au cours d'un master universitaire, pourront permettre aux futurs lauréats des concours de s'affranchir de l'exigence de la certification du niveau $\mathrm{B} 2^{3}$. Bien que ces nouvelles informations puissent soulager les étudiants qui préparent les concours pour devenir professeurs, la certification dans le cadre de l'enseignement et de l'apprentissage des langues étrangères mérite une réflexion plus approfondie ${ }^{4}$.

\section{L'évaluation des certifications des langues étrangères}

Dans ce contexte, caractérisé par l'incertitude sur le niveau de langue finalement exigé des futurs professeurs, ainsi que par «l'irrésistible ascension de l'évaluation institutionnelle» (Huver \& Springer $2011: 205$ ), notre contribution vise à offrir une démarche d'évaluation servant à comparer les certifications linguistiques.

À cet égard, il convient de rappeler le rôle du Cadre européen commun de référence pour les langues (dorénavant CECRL), dont la finalité est « de favoriser la transparence et la comparabilité des dispositifs d'apprentissage ou d'enseignement des langues et des qualifications souhaitées ou obtenues » (Goullier 2007 : 13).

Suivant l'interprétation de Bachman (1990: 22), reprise par Taillefer $\left(2007: 136^{5}\right)$, nous entendons par évaluation le « recueil systématique d'informations (quantitatives ou qualitatives) pour pouvoir interpréter et prendre des décisions ». Dans cette optique, nous avons effectué un travail de documentation et d'analyse de données permettant d'évaluer les certifications d'Espagnol Langue Étrangère (ELE) afin

\footnotetext{
${ }^{3}$ La seule condition est que ces crédits n'aient pas été obtenus par compensation avec d'autres matières ni dans des cours pour débutants et grands débutants. Par ailleurs, le site ministériel ne précise à aucun moment le nombre de crédits en langue vivante à justifier ni la relation de ces unités d'enseignement avec une compétence langagière quelconque ou avec un niveau du CECRL.

${ }^{4}$ Nous partageons les remarques de l'Association pour la Recherche en Didactique de l'Anglais et en Acquisition (<http://ardaarda.canalblog.com/archives/2011/11/index.html $>$ ) et de l'Association des Professeurs de Langues Vivantes (<http://www.aplv-languesmodernes.org/spip.php?article4175>) sur les répercussions négatives de ces décisions pour la qualification en langues des futurs enseignants.

${ }^{5}$ Selon cet auteur, la notion de certification fait allusion à toute « attestation de savoir-faire caractérisant la mise en œuvre de la compétence en langue (lire, écrire, comprendre, parler, interagir)». Cf. l'annexe de Taillefer pour la définition des termes test, certificat et diplôme (Ibid.)
} 
d'aider les enseignants et apprenants à prendre une décision raisonnée sur la certification à adopter.

Le cadre théorique de Bachman et Palmer et leur proposition d'une série de paramètres contribuant au degré d'utilité d'un test de langue (1996:17) permettent une approche novatrice de l'évaluation des certifications des langues étrangères. Face à l'opposition traditionnelle entre les critères de fiabilité et de validité, ces auteurs optent pour une approche constructive et non hiérarchisée des critères d'évaluation, en intégrant selon une même échelle six qualités complémentaires : fiabilité + validité théorique + authenticité + interactivité + impact + faisabilité ${ }^{6}$.

Une liste de questions élaborée par Bachman et Palmer (1996: 140-155) constitue un point de départ pour avancer dans toute démarche d'évaluation de l'utilité des tests. Or, comme l'indiquent ces auteurs (Ibid. : 137), il ne s'agit pas d'un inventaire fermé de questions car tout projet d'évaluation doit s'adapter aux objectifs fixés et faire éventuellement appel à d'autres aspects pertinents ${ }^{7}$. Nous allons procéder à une telle démarche en comparant l'offre des certifications d'ELE sur le marché français.

\section{Les certifications en Espagnol Langue Étrangère en France}

Selon la page <http://www.education.gouv.fr/cid56907/certificats-exiges-a-latitularisation-des-laureats-des-concours-de-professeurs-des-ecoles.html>, la liste de certifications en espagnol autres que le CLES 2 correspondant au moins au niveau B2 inclut le DCL de niveau 4 (B2) ou 5 (C1) ou le DCLEP (Diplôme de compétence en langue étrangère professionnelle) $\mathrm{B} 2$ ou $\mathrm{C}^{8}$, le BULATS avec 60 points, ainsi que le DELE de l'Institut Cervantès B2, C1 ou C2.

Nous allons étudier et comparer ces certifications linguistiques (CLES, DCLDCLEP, BULATS et DELE) en utilisant les six critères de Bachman et Palmer. Outre évaluer si ces critères d'utilité d'un test sont respectés, nous allons inclure d'autres informations utiles dans le but d'aider les candidats en espagnol et leurs enseignants à déterminer le certificat le plus approprié à leurs circonstances, intérêts et besoins particuliers pour pouvoir prendre une décision éclairée concernant

\footnotetext{
${ }^{6}$ «We propose a model of test usefulness that includes six test qualities -reliability, construct validity, authenticity, interactiveness, impact, and practicality». Pour des raisons d'espace, nous ne nous attarderons pas sur la discussion théorique de ces critères; nous renvoyons à l'analyse soigneuse et détaillée de Doucet (2001) qui définit un test utile comme « un test qui permet de faire des prédictions raisonnables tout en limitant les sources d'erreur dans la prise de mesure et les biais dans les interprétations des résultats » (Ibid. : 25).

${ }^{7}$ «You may not need to use all of these questions for a given project, and you may find that there are additional questions that need to be considered for a given project $»$.

${ }^{8}$ Depuis l'arrêté du 7 mai 2010 (JORF n ${ }^{\circ} 0108$ du 11 mai 2010), le Diplôme de compétence en langue est devenu le Diplome de compétence en langue étrangère professionnelle.
} 
leur choix de certification. Ces éléments de comparaison sont présentés dans un tableau comparatif dans l'annexe 1.

\subsection{Certificat de compétences en langues de l'enseignement supérieur (CLES)}

La création de cette certification de compétences en langues est, tout comme celle du DCL (cf. infra), " le signe d'une prise de conscience de l'importance de la reconnaissance sociale des validations institutionnelles pour les langues » (Springer 2001 : 56). Créé en 2000 et accrédité par le Ministère de l'Éducation nationale, le CLES est proposé en neuf langues vivantes, l'espagnol étant la deuxième plus demandée après l'anglais ${ }^{9}$. La fonction de ce certificat national est de permettre une évaluation externe des compétences des étudiants universitaires dans une langue étrangère dont ils ne sont pas spécialistes.

4.1.1. La notion de fiabilité renvoyant à un accord quant à la mesure ${ }^{10}$, plusieurs décisions sont mises en place pour réduire les facteurs d'erreur et pour assurer la qualité du CLES. D'une part, les concepteurs reçoivent une formation initiale et s'engagent à élaborer les sujets en respectant un cahier des charges. Par exemple, les items des épreuves de compréhension doivent permettre une correction automatisée avec des réponses simples ne nécessitant aucune interprétation du correcteur et ne doivent pas contenir d'ambigüités pouvant affecter le résultat; en ce qui concerne les épreuves de production, le concepteur précise dans le corrigé les items qui doivent figurer dans la production des candidats pour faciliter l'évaluation. Une fois élaborés, dans la mesure du possible, les sujets sont testés sur un échantillon d'étudiants pour procéder aux corrections, aux analyses et aux modifications nécessaires. Un comité de relecture agréé par la coordination nationale veille à l'harmonisation des sujets (par langue) et valide les sujets en fonction de leur adéquation avec le CECRL et de leur fidélité au cahier des charges.

De même, pour garantir la qualité des résultats, les établissements habilités à délivrer le CLES organisent la formation des évaluateurs, lesquels s'engagent à corriger les copies en respectant les critères définis par les grilles d'évaluation ${ }^{11}$. Finalement, un jury par langue se réunit pour valider les résultats des candidats.

4.1.2. Nous prenons le terme validité dans le sens de la qualité de l'interprétation des résultats ${ }^{12}$ pour expliquer «dans quelle mesure l'information recueillie donne une image exacte de la compétence des candidats en question» (Taillefer 2007 :

\footnotetext{
${ }^{9}$ Toutes les statistiques sont disponibles sur le site officiel du CLES $(<\mathrm{http}$ ://www.certificationcles.fr/>).

${ }^{10}$ Bachman et Palmer $(1996: 19):$ "Reliability is often defined as consistency of measurement ».

${ }^{11}$ Contrairement aux épreuves de compréhension, dont la réussite exige $60 \%$ des points, le candidat doit valider tous les critères pragmatiques et linguistiques des grilles de production écrite et orale pour être jugé " apte ». La gestion de la tâche, la correction grammaticale et le lexique utilisé sont évalués dans les deux épreuves. À ces critères s'ajoutent l'articulation du discours pour l'écrit et l'aisance et la phonologie pour l'interaction orale.

${ }^{12}$ Cf. Bachman et Palmer (1996:21).
} 
136). S'appuyant sur le CECRL, ce certificat se décline en trois degrés de qualification (CLES 1, 2 et 3 ) et permet de valider la compétence des candidats en espagnol à partir des descripteurs des niveaux B1, B2 et $\mathrm{C} 1$ du Conseil de 1'Europe (2001). Afin d'obtenir le niveau B2 en espagnol, le candidat doit montrer que son usage de la langue correspond à celui d'un utilisateur indépendant.

4.1.3. L'ensemble des épreuves du CLES se déroulent sous la forme actionnelle d'un scénario et de la résolution d'un problème. L'authenticité ${ }^{13} \mathrm{du}$ CLES est visée au moyen d'une mise en situation vraisemblable et de l'accomplissement d'une mission ancrée dans le monde réel. La définition d'un rôle, des interlocuteurs et du destinataire final assure la crédibilité (validité faciale) de ce certificat et montre l'adéquation entre les tâches du test et l'emploi réel de la langue en situation horstest.

Le CLES se présentant sous forme de scénario, toutes les tâches à effectuer par les candidats sont liées et s'enchaînent. Les tâches de compréhension et d'expression portent sur la même thématique et les informations contenues dans les documents audiovisuels et écrits (toujours authentiques) deviennent indispensables pour les macro-tâches de production écrite et orale.

4.1.4. Ce certificat est proposé aux étudiants de l'enseignement supérieur non inscrits dans une filière linguistique et notamment aux candidats aux concours de recrutement du personnel enseignant des premier et second degrés. S'agissant d'une certification pour spécialistes d'autres disciplines que les langues, le CLES ne demande pas de connaissances spécifiques préalables. Ayant participé en tant qu'évaluateur du CLES 2, nous avons constaté une incidence des caractéristiques personnelles et des réactions affectives des candidats sur la réussite de l'interaction orale qui dépend parfois de leur goût pour jouer le rôle assigné ${ }^{14}$.

4.1.5. Quel est l'impact de ce certificat? Tout d'abord, le CLES 2 est conçu afin de permettre de valider les compétences de communication en langue étrangère des futurs enseignants en France. De même, s'agissant d'une certification en accord avec le CECRL, le CLES constitue un passeport pour la mobilité des étudiants. En définitive, ce certificat devient un outil très intéressant pour la poursuite des études universitaires et pour l'avenir professionnel des candidats.

Par ailleurs, les formations des concepteurs et des examinateurs CLES sur le plan national facilitent l'accord des enseignants de langues sur les critères d'évaluation choisis et les décisions prises. Cependant, malgré cet effet positif, on peut craindre pour le système éducatif d'éventuelles conséquences négatives d'une généralisation des enseignements universitaires de langues conditionnés à la préparation du

\footnotetext{
${ }^{13}$ Bachman et Palmer (1996: 23) définissent ce critère «as the degree of correspondence of the characteristics of a given language test task to the features of a TLU task».

${ }^{14} \mathrm{Cf}$. Bachman et Palmer (1996: 25) : "We define interactiveness as the extent and type of involvement of the test taker's individual characteristics in accomplishing a test task ».
} 
CLES. Comme l'indique Buck (2007 : 94) : «l'emploi du scénario comme démarche d'apprentissage constitue une familiarisation avec les critères et le mode d'évaluation » qui favorise la réussite des candidats. Or, il faut éviter que les cours deviennent un entraînement à un format concret, car « enseigner en vue d'un examen », n'est « pas nécessairement cohérent, pertinent ou valide» (Taillefer 2007 : $134)^{15}$. Ainsi, le site CLES ne publie pas d'annales afin d'éviter le «bachotage » pour se préparer à l'examen; un seul CLES 2 en ligne en espagnol $(<\mathrm{http}: / /$ www.certification-cles.fr/ $>)$ permet aux candidats de se familiariser avec ce certificat.

4.1.6. Quant aux ressources permettant d'assurer la faisabilité ${ }^{16}$ du CLES 2, ce certificat requiert la participation de plusieurs types d'intervenants : coordonnateurs des pôles académiques, concepteurs et relecteurs des sujets, surveillants, correcteurs des épreuves écrites et orales, et membres des jurys de validation des résultats.

Le temps de passation du CLES 2 est de 3 heures. L'examen commence par l'épreuve de compréhension orale (30 minutes), au cours de laquelle le candidat répond à des questions portant sur deux ou trois documents audio et / ou vidéo. Puis, pour les épreuves de compréhension et de production écrites, les étudiants gèrent le temps qui leur est accordé ( 2 heures et quart) afin de lire plusieurs textes, répondre aux questions de compréhension et rédiger une synthèse (250-300 mots). Enfin, les candidats passent en binôme ou en trinôme l'épreuve d'interaction orale (10'-15 minutes).

Par ailleurs, tous les candidats passent les examens dans les centres habilités, dans lesquels une salle multimédia est prévue afin d'assurer l'écoute et le visionnage des documents de la compréhension orale et de faire passer les épreuves écrites; ensuite, d'autres salles sont utilisées pour l'interaction ${ }^{17}$. S'agissant d'une certification d'État, le premier passage du CLES est gratuit pour les étudiants et, en cas d'échec, les frais d'inscription à une session postérieure sont d'environ $25 €^{18}$.

\footnotetext{
15 Dans notre cas, s'agissant de cours pour les futurs enseignants, nous conseillons une formation promouvant la réflexion sur ce que signifie apprendre une langue étrangère et facilitant le travail en cours de langue et/ou en autoformation sur les compétences langagières en espagnol.

${ }^{16}$ Nous étudions les ressources humaines nécessaires pour la conception, le pilotage, l'administration et la correction des examens, ainsi que les considérations matérielles de passation (temps, locaux, équipement, coût et dates).

${ }^{17}$ Chaque centre habilité fixe le nombre des sessions qu'il organise et établit le calendrier des épreuves. En Lorraine, nos candidats d'espagnol passent les épreuves à Nancy une fois par an, au mois de mai.

${ }^{18}$ L'aspect financier peut constituer un atout du CLES par rapport aux certifications linguistiques payantes, telles que le DCL, le BULATS ou le DELE.
} 


\subsection{Diplôme de compétence en langue (DCL) - Diplôme de compétence en langue étrangère professionnelle (DCLEP)}

Cette double étiquette fait allusion à un diplôme accrédité par le Ministère de l'Éducation nationale en France servant à évaluer la capacité à utiliser une langue étrangère en situation professionnelle. Jusqu'en 2010, le DCL existait en quatre langues (allemand, anglais, espagnol et italien). Depuis 2011, ce diplôme s'est ouvert à d'autres langues et en concerne actuellement treize.

4.2.1. L'élaboration des sujets est de la responsabilité de concepteurs agréés ayant suivi une formation et participé au préalable en tant qu'examinateurs, ce qui vise à assurer la fiabilité du DCL. Une commission nationale relit et valide les sujets sur la base d'un cahier des charges. La correction des tests est assurée par des examinateurs habilités : universitaires et réseaux Greta (Groupements d'établissements publics locaux d'enseignement), à partir de grilles critériées permettant une évaluation détaillée des épreuves ${ }^{19}$. Un jury composé d'inspecteurs, d'universitaires et d'examinateurs propose enfin la délivrance ou non du diplôme, avec la mention du niveau du CECRL ${ }^{20}$.

4.2.2. Ce diplôme correspond à l'analyse des besoins professionnels et c'est la performance réalisée (efficacité de la communication pour mener à bien la mission et qualité linguistique de la prestation) qui détermine l'obtention d'un niveau du CECRL (A2, B1.1., B1.2, B2 ou C1). La validité du DCL est assurée par la qualité des interprétations des résultats : le diplôme signé par le recteur de l'académie atteste du niveau de compétence en langue professionnelle du candidat et contient au verso un descriptif des compétences validées. Comme l'indique Springer (2001: 57 ), « [c]ette validation possède tous les atouts pour certifier qu'une personne est en mesure de faire face à l'ensemble des situations de communication en milieu professionnel ». Pour valider le niveau B2 en espagnol (degré 4, opérationnalité efficace), un candidat doit être capable de s'exprimer à l'oral et à l'écrit en utilisant un lexique varié et en démontrant une bonne maîtrise du code (quelques erreurs étant permises). De même, s'agissant d'un diplôme à visée professionnelle, le candidat doit justifier de qualités d'initiative et d'autonomie dans le traitement, l'organisation et la hiérarchisation d'informations, d'adaptation aux situations imprévisibles, ainsi que d'une autonomie lui permettant de participer avec efficacité à

\footnotetext{
${ }^{19}$ Un exemple de profil détaillé de l'évaluation d'un candidat au DCL, consultable sur le site $<$ http://www.education.gouv.fr/cid55748/le-diplome-de-competence-en-langue-dcl.html $>$, montre la combinaison de critères pragmatiques d'évaluation (qualité de la communication, capacité d'adaptation à l'environnement professionnel, respect de la consigne, degré d'accomplissement de la mission confiée, etc.) et de critères linguistiques, portant sur les niveaux phonologique (sons, accentuation, rythme et intonation), lexical (richesse et variété), morphosyntaxique (structuration de phrase, morphologie verbale, accords, etc.) et graphique (ponctuation et orthographe).

${ }^{20}$ Le niveau est établi en cohérence avec les descripteurs du référentiel de certification figurant en annexe de l'arrêté du 7 mai 2010.
} 
des échanges en espagnol. De plus, tout candidat obtient sur Internet un profil explicatif de sa prestation.

4.2.3. Un des points forts du DCL est qu'il donne une place fondamentale au concept d'utilisation de la langue espagnole dans des situations hors test (vie réelle, secteur professionnel). L'évaluation portant sur la capacité à employer la langue dans une situation aussi proche que possible de la réalité du travail, ce diplôme indique avec quel degré de compétence la personne est en mesure d'utiliser l'espagnol en tant que langue de communication usuelle et professionnelle. Ce diplôme «revendique sa particularité fonctionnelle et interactionnelle (il s'agit d'un scénario qui simule un rôle professionnel)» (Springer $2001: 57$ ). La forme actionnelle d'un scénario et la simulation de situations de communication réelles justifient l'authenticité du DCL. S'appuyant sur des documents authentiques, le candidat fait face à un continuum de cinq phases successives, intégrant des tâches liées entre elles en adéquation avec les tâches qu'il peut rencontrer hors test (dans la réalité professionnelle).

Le DCL se présente sous forme de scénario comportant l'ensemble des compétences langagières (compréhension de l'écrit et de l'oral, production écrite et orale, et communication interactive) dans le cadre d'une mission à réaliser. Le DCL évalue de façon positive la compétence en langue en référence au degré d'opérationnalité dans l'accomplissement de la mission.

4.2.4. La mention «langue étrangère professionnelle » ayant été ajoutée, le DCL s'adresse aux adultes (employés, stagiaires, demandeurs d'emploi, étudiants, etc.) désirant faire reconnaître en France leur capacité à utiliser l'espagnol comme langue de communication opérationnelle ${ }^{21}$. Cet examen est ouvert à tout adulte sans condition de nationalité et d'activités professionnelles préalables. Par ailleurs, aucune formation en langues n'est demandée pour passer l'examen.

4.2.5. Cette certification permet d'évaluer la compétence en langue opérationnelle en milieu professionnel. Ainsi, le DCL facilite la validation de compétences en espagnol dans le cadre d'une démarche de développement personnel, d'insertion, d'évolution ou de mobilité professionnelle des candidats. La création de ce diplôme a eu un impact très positif sur les entreprises souhaitant mesurer la compétence en langue de leurs collaborateurs dans le cadre des recrutements ou des évaluations en fin de formation. La préparation au DCL s'est également insérée dans le système éducatif, notamment avec des formations aux espaces langues des Greta ${ }^{22}$.

\footnotetext{
${ }^{21}$ La forme du scénario professionnel du DCL peut entraîner des réactions affectives positives des candidats : "Le fait que la mission fasse appel aux compétences professionnelles met clairement l'accent sur la langue comme outil de travail, d'où l'accroissement de la motivation des apprenants » (Buck 2007 : 94).

${ }^{22}$ De même, le CNED offre des formations pour préparer le DCL. Par ailleurs, un exemple d'examen aidant à comprendre le déroulement des épreuves est offert en ligne aux candidats en espagnol : $<$ http://www.education.gouv.fr/cid55748/le-diplome-de-competence-en-langue-dcl.html>.
} 
4.2.6. La faisabilité du DCL dépend de la participation de plusieurs acteurs, comme les responsables académiques en charge de l'organisation de l'examen, la commission nationale de coordination, les concepteurs et les examinateurs habilités, le personnel surveillant et les inspecteurs faisant partie des jurys.

Le temps de passation de l'examen est de 2 heures et 30 minutes. D'abord, le candidat dispose d'une heure et demie pour comprendre la mise en situation, lire environ cinq textes, écouter deux enregistrements sonores ou audiovisuels, répondre à une série d'items sous forme de prise de notes et préparer la simulation d'un entretien téléphonique. Cet entretien (avec un expert en présence du candidat ou à distance) sert à évaluer les compétences d'expression et d'interaction orales et dure 20 minutes. La dernière épreuve est une synthèse écrite (40 minutes) permettant d'exposer une solution au problème abordé.

Le DCL se passe dans des centres agréés (Greta et universités), avec des salles équipées pour l'écoute des documents sonores, pour l'entretien téléphonique et pour les épreuves écrites. Le ministre chargé de l'éducation arrête le calendrier des sessions d'examen ${ }^{23}$, dont les frais d'inscription s'élèvent à $100 €$.

\subsection{Business Language Testing Service (BULATS)}

Comme l'indique le site $<$ http://www.bulats.org/>, ce test est conçu et géré par l'Université de Cambridge ESOL, en collaboration avec des institutions spécialisées en évaluation des compétences : l'Alliance Française pour le français, le Goethe-Institut pour l'allemand et l'Université de Salamanque pour l'espagnol.

4.3.1. Le BULATS évalue la capacité à utiliser une langue étrangère dans un contexte professionnel. Ce test, développé et géré par un consortium international de concepteurs membres de l'ALTE (Association of Language Testers in Europe), est conforme à un certain nombre de contrôles et de normes de qualité. Ainsi, de nombreuses expérimentations sont effectuées pour garantir la fiabilité du BULATS $^{24}$.

À la différence du CLES ou du DCL, le test standard BULATS présente les résultats en termes de score (notation globale de 0 à 100 points et score partiel des sousépreuves) ; dans sa version informatisée, le BULATS est un test auto-adaptatif qui cible les compétences du candidat avec rapidité en utilisant des techniques d'évaluation modulables, ce qui permet une correction automatique des résultats ${ }^{25}$.

\footnotetext{
${ }^{23}$ Le DCL en espagnol est limité à deux sessions par an (mai/juin et novembre/décembre) et n'est malheureusement pas ouvert dans toutes les académies françaises.

${ }^{24}$ Le pretesting constitue une partie essentielle du processus de conception des examens. Il permet aux apprenants de se familiariser avec le test grâce au passage gratuit de modèles d'examen abrégés qui aident les concepteurs à prendre des décisions pour ajuster les contenus des épreuves.

${ }^{25}$ La connaissance rapide des résultats fait du BULATS informatisé une certification de plus en plus appréciée par les candidats.
} 
Quant aux épreuves d'expression (écrite et orale), la correction est assurée par deux experts qui accordent une note globale de 0 à 5 d'après l'échelle de six niveaux de l'ALTE, affinée par un signe $(+,=,-)$ précisant si la performance du candidat se situe dans la partie supérieure, moyenne ou inférieure du niveau attribué ${ }^{26}$.

4.3.2. Contrairement à d'autres outils d'évaluation comme le DELE ou le CLES, le BULATS ne comporte pas pas de notion d'échec ou de réussite. Ce test permet de connaître le niveau de compétence atteint sur une échelle fondée sur les niveaux établis par l'ALTE (0-5) et le CECRL (A1-C2). Au verso du diplôme, un rapport expliquant le score permet d'interpréter les résultats en termes de compétences dans le milieu des affaires ; ainsi, le BULATS décrit ce que le candidat saurait faire dans un contexte professionnel.

4.3.3. Le BULATS vise, comme le DCL, à tester la compétence en langue étrangère dans des situations authentiques. Ainsi, à partir des documents couvrant des domaines proches de la réalité professionnelle ${ }^{27}$, le test tente d'évaluer les compétences linguistiques requises sur le lieu de travail.

Or, contrairement au CLES et au DCL, le BULATS n'est pas conçu sous la forme d'un scénario de tâches intégrées, mais composé de trois tests indépendants (compréhension, expression écrite et expression orale). Dans le BULATS généralement demandé pour certifier les compétences en langue (le test de compréhension), le candidat ne produit pas d'échantillon linguistique, ce qui constitue un gros problème quant à l'authenticité du test. De même, le type d'exercices du BULATS standard (questions à choix multiple, textes et phrases à trous) dévoile une approche plutôt traditionnelle visant notamment à mesurer la compétence grammaticale des candidats.

4.3.4. Quant à l'interactivité du BULATS, ce test prend en compte les caractéristiques des candidats : un public adulte international (employés, stagiaires, demandeurs d'emploi, etc.) ayant besoin d'attester de ses compétences en communication linguistique dans le milieu des affaires. Tout comme le DCL, le BULATS fait appel aux compétences professionnelles et le recours à la langue en tant qu'outil de travail augmente la motivation et les réactions affectives positives des candidats.

${ }^{26}$ Pour l'expression écrite, chaque évaluateur vérifie : correction linguistique, registre, organisation, présentation et précision. Pour l'épreuve orale (enregistrée pour évaluation d'un deuxième expert), les critères examinés sont : registre et ressources de langue, précision communicative, prononciation, interaction et gestion du discours. Pour assurer la fiabilité et la stabilité des corrections, dans le cas (peu habituel) d'une disparité entre les notes, les épreuves d'expression sont évaluées par un troisième expert.

${ }^{27}$ Parmi les thèmes et situations abordés dans le test BULATS, citons : la demande d'informations et les tâches quotidiennes dans un contexte professionnel (organisation des rendez-vous, planification d'événements, service clientèle, achat et vente de produits, réservations, rapports, réclamations, etc.), ainsi que les centres d'intérêt liés au monde du travail (gestion, marketing, etc.). Pour plus de renseignements concernant les thématiques, la composition de chacune des parties du test ou les types de questions, un guide est disponible en ligne : <http://www.bulats.org/Bulats/Handbooks.html>. 
Malgré son profil professionnel et sa version informatisée, la passation du test ne requiert pas d'expérience préalable dans le domaine commercial ni de connaissances spécifiques en informatique ${ }^{28}$.

4.3.5. Le BULATS participe à plusieurs projets internationaux visant «à définir des niveaux de compétences cohérents pour l'enseignement et l'évaluation des langues étrangères $»^{29}$.

Ce test est employé par de nombreux établissements éducatifs pour diagnostiquer les besoins des apprenants en termes de formation linguistique ${ }^{30}$. D'autre part, le test BULATS informatisé permet aux entreprises une évaluation rapide de la compétence en langue étrangère de leurs employés et une prise de décision rapide dans le processus de recrutement.

Enfin, s'agissant d'un test de référence dans l'évaluation de l'espagnol en milieu professionnel, l'obtention d'un bon score pourrait avantager un candidat sur le marché du travail, car, tout comme le DELE (infra), le BULATS jouit d'une forte reconnaissance internationale.

4.3.6. Ce test prévoit trois épreuves pouvant être effectuées de façon indépendante ou combinée en fonction des besoins d'évaluation des candidats. D'une part, un examen distribué en version papier (appelé « test standard ») ou en format informatisé (sur CD-ROM ou en ligne) permet d'évaluer les compétences de compréhension orale et écrite, ainsi que la grammaire et le vocabulaire. D'autre part, deux tests complémentaires servent à passer l'expression écrite et orale.

Le test standard complet, dans sa version papier, dure 110 minutes et nécessite du personnel surveillant et des examinateurs agréés. Dans sa version informatisée, le BULATS est un test adaptatif : le logiciel règle le niveau de difficulté des questions sur la base des réponses précédentes; même si ce test peut être effectué en ligne, un agent BULATS ou un enseignant surveillant veille au bon déroulement de l'épreuve, dont la durée maximale de passation est de 75 à 85 minutes. Par ailleurs, le test d'expression écrite en espagnol (rédaction d'un message et écriture d'un rapport ou d'une lettre) dure trois quarts d'heure et est évalué par deux examinateurs. Enfin, l'autre test complémentaire (expression orale) dure 12 minutes et comporte trois parties : informations personnelles, court exposé sur un thème relatif au monde du travail et interaction avec un expert sous la forme d'un jeu de rôle.

\footnotetext{
${ }^{28}$ Le BULATS (<http://www.bulats.org/Bulats/Sample-tests.html $>$ ) présente un exemple du test standard papier en espagnol, avec un corrigé facilitant l'autoévaluation. Quant à la version informatisée, des démonstrations en ligne

( $<$ https://bulatsdt.bulatsonline.org/appTest/demoTestModePage.html $>)$ avec des tutoriels aident à comprendre les consignes du test.

${ }^{29} \mathrm{Cf}$. $<$ http://www.bulats.org/learning-resources/handbooks $>$.

${ }^{30}$ Malgré la mise en place des formations facilitant le passage du BULATS, le marché éditorial n'a pas encore prévu, à notre connaissance, de manuels spécifiques de préparation au BULATS en espagnol.
} 
Il n'est pas nécessaire d'avoir des locaux spécifiques pour faire passer le BULATS (uniquement un ordinateur pour la version informatisée et un lecteur CD pour le test standard de compréhension). Contrairement aux autres certifications, le BULATS a l'avantage de pouvoir s'effectuer quand et où l'on veut (il peut se faire directement dans l'entreprise). Pour passer un test en France, il n'y a pas besoin d'attendre les dates d'un calendrier officiel de sessions: il suffit de contacter l'agent BULATS le plus proche, dont les coordonnées sont en ligne $(<\mathrm{http}: / /$ www.bulats.org/All-agents/france.html $>)$, et de convenir d'une date et d'une modalité d'examen.

Quant aux tarifs BULATS, le test de compréhension peut coûter entre quarante et soixante euros. À cette somme, il faut ajouter environ cinquante euros pour chacune des épreuves d'expression (écrite et orale).

\subsection{Diploma de Español como Lengua Extranjera (DELE)}

L'Institut Cervantès, organisme créé en 1991 pour diffuser la culture des pays hispanophones et promouvoir l'enseignement de la langue espagnole, est responsable de l'administration du DELE.

4.4.1. Le DELE (<http://diplomas.cervantes.es/>) est un diplôme officiel attestant le degré de compétence en langue espagnole et reconnu par le Ministère de l'Éducation et des Sciences d'Espagne. La conception des examens DELE par l'Université de Salamanque en adéquation avec les directives du Cadre européen facilite la mesure du niveau des candidats. Par ailleurs, la formation spécifique reçue par les examinateurs afin de les familiariser aux descripteurs de chaque niveau du CECRL et à l'utilisation précise et efficace des grilles d'évaluation vise à assurer la qualité et la fiabilité de ce diplôme.

Fondé sur un principe de régularité, l'examen DELE a lieu à la même date dans tous les centres agréés d'un même pays. De plus, afin d'augmenter la fiabilité interne des évaluations, les épreuves d'expression orale et écrite ne sont pas évaluées par un seul examinateur (cas du CLES et DCL) mais par deux experts, formés au préalable aux critères d'évaluation pour assurer un accord dans la notation des épreuves et pour réduire tout facteur d'erreur dans l'évaluation d'un même candidat $^{31}$. Face aux grilles qualitatives limitant l'évaluation à un jugement global du type validé/non validé, l'accord par rapport aux critères d'évaluation vise à assurer une mesure précise de la performance de chaque candidat ${ }^{32}$.

\footnotetext{
${ }^{31}$ Par ailleurs, la feuille de réponses pour les épreuves de compréhension, de grammaire et de vocabulaire est corrigée automatiquement par ordinateur. Pour le DELE B2, tout candidat est noté sur 100 points : pour être « apte», il doit obtenir $70 \%$ des points dans chaque sous-groupe d'examen.

32 Cf. Doucet (2001: 16) : «Lorsque l'on effectue des évaluations, on reste dans le domaine de l'impression. Une évaluation critériée amène à réduire la zone à l'intérieur de laquelle nos observations sont le fruit de nos impressions ».
} 
4.4.2. Contrairement aux centres produisant des certifications BULATS et DCL, l'Institut Cervantès, membre de l'ALTE, organise des examens pour chacun des six niveaux du CECRL (A1-C2). Ainsi, les diplômes DELE valident et certifient un niveau de performance attendu en référence aux descripteurs du CECRL, avec un score global et des résultats partiels dans chacune des épreuves ${ }^{33}$.

4.4.3. À la différence du DCL ou du CLES, les examens DELE ne sont pas conçus sous la forme actionnelle d'un scénario. Dans le DELE B2, chaque candidat est évalué à partir de cinq épreuves différenciées (compréhension écrite, expression écrite, grammaire et vocabulaire, compréhension orale et expression orale). Suivant une approche communicative, le DELE B2 vise une compétence suffisante en espagnol pour aborder des situations courantes de la vie quotidienne, ne demandant pas l'usage d'une langue spécialisée.

4.4.4. Avec un réseau de plus de 700 centres d'examen, les diplômes de l'Institut Cervantès s'adressent à un public international, généralement des adultes ou de jeunes adultes, désirant attester d'un niveau de maîtrise de la langue espagnole qu'ils puissent faire valoir sur le marché du travail. Généralement, les candidats au DELE ont suivi une formation en espagnol, grâce aux possibilités de préparation dans les centres de l'Institut Cervantès et dans leurs institutions partenaires ${ }^{34}$.

4.4.5. Le degré de compétence en langue espagnole validé par un diplôme DELE est reconnu au niveau mondial. Contrairement à ce qui se passe pour les certifications nationales (DCL, CLES), le réseau de centres dans plus de cent pays assure la diffusion internationale du DELE.

De plus, accrédité par le Ministère de l'Éducation et des Sciences d'Espagne, le DELE a eu un grand impact sur le système éducatif, notamment pour l'enseignement et la diffusion de l'Espagnol Langue Étrangère dans le domaine hispanique. Ainsi, de nombreuses formations initiales et approfondies des enseignants d'ELE sont ouvertes tous les ans dans les centres officiels et les organismes partenaires de l'Institut Cervantès. Par ailleurs, l'expansion des diplômes DELE explique la publication croissante de manuels destinés à la préparation aux différents examens DELE ${ }^{35}$.

4.4.6. La collaboration de l'Université de Salamanque (personnel chargé de la conception et de la correction des épreuves écrites) et du réseau de centres de l'Institut Cervantès (personnel disponible pour l'administration et la surveillance des examens, ainsi que des examinateurs pour l'évaluation de l'expression orale) permet d'assurer la faisabilité des diplômes DELE.

\footnotetext{
${ }^{33}$ Pour l'interprétation des capacités mesurées au B2, on peut voir les indications sur le site: $<$ http://diplomas.cervantes.es/informacion/niveles/nivel_b2.html $>$.

${ }^{34} \mathrm{Par}$ ailleurs, le site $<\mathrm{http}$ ://diplomas.cervantes.es/> offre des ressources pour les enseignants et apprenants d'ELE et présente des annales des examens du DELE.

${ }^{35}$ Par exemple, voir Alzugaray, Barrios et Hernández (2009) et García Muruais et al. (2010).
} 
Le temps de passation du DELE B2 dépasse celui des autres certifications examinées. Cet examen débute par la compréhension écrite (quatre textes avec QCM) et l'expression écrite (lettre et rédaction de 150-200 mots chacune) d'une durée de 2 heures. Après une pause, dans la partie de compréhension auditive (30 minutes), les candidats répondent par écrit à des questions concernant des documents sonores. Ensuite, le candidat réalise une épreuve de grammaire et de vocabulaire (1 heure) avec des questions à choix multiple. Avec un décalage temporel, les candidats sont convoqués pour l'épreuve d'expression orale (15 minutes de préparation et le même temps de passation) qui s'effectue devant deux évaluateurs (l'un jouant le rôle d'intervieweur et l'autre de correcteur) : le candidat décrit d'abord quelques vignettes illustrant des situations de communication quotidienne en espagnol, puis expose un thème préparé et enfin interagit avec un expert sur la même thématique.

Les frais d'inscriptions au DELE B2 en France s'élèvent à 142 euros et il y a deux sessions d'examen par an (mai et novembre) se déroulant dans un réseau de centres répartis sur l'ensemble du territoire français ${ }^{36}$.

\section{Conclusion}

L'offre des certifications en France pour attester du niveau B2 en espagnol langue étrangère est variée. D'une part, les candidats peuvent choisir une certification d'état comme le CLES, dont l'examen est gratuit pour les étudiants universitaires. Conçu sous la forme d'un scénario et dans le cadre d'une mission à accomplir, l'obtention de ce certificat exige la validation de toutes les épreuves de compréhension et de production, sans compensation possible entre les résultats. L'autre certification nationale (DCL) s'adresse à un public d'adultes et vise à évaluer la compétence en langue opérationnelle des candidats en milieu professionnel, ce qui assure une adéquation entre les tâches dans le test et en situation hors-test (monde du travail) ; malheureusement, le DCL en espagnol n'est pas ouvert dans toutes les académies de France. Par ailleurs, la flexibilité des modalités de passation du test BULATS par rapport aux autres certifications d'espagnol, ainsi que la connaissance rapide des résultats du test informatisé peuvent décider certains candidats à choisir ce test. En effet, malgré son profil professionnel, le BULATS standard est composé d'épreuves traditionnelles (QCM, exercices lacunaires) et ne demande pas d'échantillon produit par les candidats. Le diplôme de l'Institut Cervantès (DELE), reconnu par le Ministère de l'Éducation et des Sciences d'Espagne, jouit d'une très grande renommée internationale grâce à un réseau de centres répartis dans le monde; la correction automatique des sujets, ainsi que l'évaluation par deux experts des épreuves de production visent à réduire tout facteur d'erreur dans la me-

\footnotetext{
${ }^{36}$ La liste détaillée des centres d'examen et leurs coordonnées est disponible à l'adresse suivante : $<\mathrm{http}$ ://diplomas.cervantes.es/informacion/centros_examen_espanol.html?cod_pais=250>.
} 
sure des compétences des candidats, même si l'épreuve de grammaire et de vocabulaire du niveau B2 est fondée sur une approche traditionnelle ${ }^{37}$.

Parler d'une certification idéale semble difficile, car certaines caractéristiques peuvent être perçues différemment par les enseignants et les apprenants en espagnol. Évidemment, la décision sur le choix d'une certification dépend du candidat en fonction de ses besoins personnels et/ou professionnels: certification nationale (CLES, DCL) ou internationale (BULATS, DELE), visée professionnelle (DCL, BULATS) ou plutôt académique (CLES, DELE), etc.

Notre but dans cet article n'est pas de plaider en faveur d'une certification donnée, mais d'analyser des critères permettant d'avancer dans une démarche d'évaluation de ces produits. Dans ce but, les lecteurs trouveront en annexe un tableau récapitulatif offrant une comparaison critériée aussi complète que possible entre les quatre certifications examinées (CLES, DCL, BULATS et DELE). À partir des critères d'évaluation de l'utilité des tests de langue (fiabilité, validité, authenticité, interactivité, impact et faisabilité), nous essayons de présenter les ressemblances et les différences entre ces quatre certifications de manière à aider les enseignants et les apprenants en espagnol à faire leur choix en toute connaissance de cause.

\section{Bibliographie}

Alzugaray, P., M.J. Barrios \& C. Hernández. 2009. Preparación al Diploma de Español Nivel Intermedio B2. Madrid : Edelsa.

Association des Professeurs de Langues Vivantes. 2011. Validation de compétences en langues sans le CLES 2 : on ne saurait en rester là, en ligne, mis en ligne le 11/11/2011, consulté le 21/09/2012 <http://www.aplvlanguesmodernes.org/spip.php?article4175>.

Association pour la Recherche en Didactique de l'Anglais et en Acquisition. 2011. Le CLES 2: retour à la case départ, en ligne, mis en ligne le 21/11/2011, consulté le $<$ http://ardaarda.canalblog.com/archives/2011/11/index.html $>$.

Bachman, L. 1990. Fundamental considerations in language testing. Oxford: Oxford University Press.

Bachman, L.F. \& A.S. Palmer. 1996. Language testing in practice. Oxford: Oxford University Press.

Buck, J. 2007. « Le scénario : une réponse à l'application du CECRL ». Cahiers de $l^{\prime} A P L I U T$, vol. XXVI, $n^{\circ} 2: 81-102$.

Conseil de l'Europe. 2001. Cadre européen commun de référence pour les langues : apprendre, enseigner, évaluer. Paris : Didier.

Doucet, P. 2001. " Pour un test utile». ASp, $n^{\circ} 34: 13-33$.

\footnotetext{
${ }^{37}$ Nous faisons allusion au format actuel car, à partir de 2013, de nouvelles épreuves pour le DELE B2 sont prévues.
} 
García Muruais, T. et al. 2010. El cronómetro. Manual de preparación al DELE. B2. Madrid : Edinumen.

Goullier, F. 2007. « Le Cadre européen commun de référence pour les langues, instrument de normalisation ou document instrumentalisé pour une normalisation de l'enseignement et de l'évaluation? ». Cahiers de l'APLIUT, vol. XXVI, $n^{\circ} 2: 12-22$.

Huver, E. \& C. Springer. 2011. L'évaluation en langues. Paris : Didier.

Instituto Cervantes. 2012. Diplomas de Español como Lengua Extranjera, en ligne, consulté le 21/09/2012<http://diplomas.cervantes.es/>.

Ministère de l'Éducation nationale. 2012a. Certificats exigés à la titularisation des lauréats des concours de professeurs des écoles, en ligne, mise à jour en septembre 2012, consulté le 21/09/2012 [ $<$ http://www.education.gouv.fr/cid56907/certificats-exiges-a-la-titularisationdes-laureats-des-concours-de-professeurs-des-ecoles.html $>$ ]

Ministère de l'Éducation nationale. 2012b. Diplôme de compétence en langue, en ligne, mise à jour en septembre 2012, consulté le 21/09/2012 $<$ http://www.education.gouv.fr/cid55748/le-diplome-de-competence-en-languedcl.html>.

Ministère de l'Éducation nationale. 2010a. « Arrêté du 31 mai 2010 fixant les titres, diplômes, certificats, attestations ou qualifications équivalentes attestant des compétences en langues de l'enseignement supérieur et en informatique et internet exigés de candidats ayant subi avec succès les épreuves des concours de recrutement de personnels enseignants des premier et second degrés et de personnels d'éducation relevant du ministre chargé de l'éducation nationale ». Journal Officiel de la République Française, $\mathrm{n}^{\circ} 0139$ du 18/06/2010, texte $\mathrm{n}^{\mathrm{o}} 18$.

Ministère de l'Éducation nationale. 2010b. «Arrêté du 7 mai 2010 relatif au diplôme de compétence en langue étrangère professionnelle ». Journal Officiel de la République Française, ${ }^{\circ} 0108$ du 11/05/2011, texte $n^{\circ} 31$.

Ministère de l'Enseignement supérieur et de la recherche. 2012. Certificat de compétences en langues de l'enseignement supérieur, en ligne, consulté le 21/09/2012<http://www.certification-cles.fr/>.

Springer, C. 2001. « Diagnostic, bilan de compétences, certification : les nouveaux habits de l'évaluation». Les Langues Modernes, $n^{\circ}$ 2: 48-60.

Taillefer, G. 2007. " Normes et normalisation dans l'enseignement des langues » Compte rendu de la table ronde sur les certifications (2 juin 2006)». Cahiers de l'APLIUT, vol. XXVI, $n^{\circ} 2: 133-139$.

University of Cambridge ESOL Examinations. 2012. Business Language Testing Service, en ligne, consulté le 21/09/2012<http://www.bulats.org/>. 
Annexe 1. Tableau comparatif des quatre certifications d'ELE en France en 2012

\begin{tabular}{|c|c|c|c|c|c|}
\hline Fiabilité & $\begin{array}{l}\text { Acc' rd entre les mesu- } \\
\text { res, réducti`n de fac- } \\
\text { teurs d'erreur, qualité } \\
\text { des résultats }\end{array}$ & $\begin{array}{l}\text { Référentiel CECRL, } \\
\text { f' rmati` } n \text { de c` ncepteurs, } \text { relecture des sujets, exa- } \\
\text { minateurs habilités, c` r- } \\
\text { recti`n aut' matisée de } \\
\text { CO et CE, grilles PE et } \\
\text { IO, jurys }\end{array}$ & 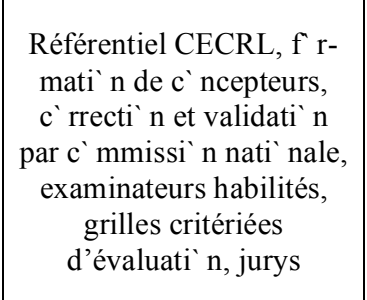 & $\begin{array}{l}\text { Référentiel ALTE et } \\
\text { CECRL, c' ntrôles de } \\
\text { qualité (pretesting), test } \\
\text { adaptatif (c` rrecti` n } \\
\text { aut` matique), grilles } \\
\text { critériées p' ur EE et EO, } \\
\text { évaluati` n par } 2 \text { experts } \\
\text { (3ème si nécessaire) }\end{array}$ & $\begin{array}{l}\text { Référentiel CECRL, f'r- } \\
\text { mati` ns d'examinateurs, } \\
\text { c' rrecti` n aut' matique par } \\
\text { `rdinateur, grilles crité- } \\
\text { riées p`ur EE et EO, éva- } \\
\text { luati` n par } 2 \text { experts (3ème } \\
\text { si nécessaire) }\end{array}$ \\
\hline Validité & $\begin{array}{l}\text { Qualité des interpréta- } \\
\text { ti` ns }\end{array}$ & $\begin{array}{c}\text { Adéquati` n avec les } \\
\text { descripteurs et les ni- } \\
\text { veaux B1-C1 du CECRL. } \\
\text { Résultat gl` bal et par } \\
\text { c`mpétence }\end{array}$ & $\begin{array}{l}\text { Bes`ins pr` fessi` nnels. } \\
\text { Résultat gl` bal par niveau } \\
\text { (A2-C1), descriptif et } \\
\text { pr` fil des c`mpétences }\end{array}$ & $\begin{array}{l}\text { Langue en c` ntexte pr` - } \\
\text { fessi`nnel. Sc` re }(1-100) \\
\text { et rapp 'rt explicatif }(0-5 \\
\text { ALTE et A1-C2 CECRL) }\end{array}$ & $\begin{array}{l}\text { Adéquati` } \mathrm{n} \text { avec les ni- } \\
\text { veaux du CECRL (A1- } \\
\text { C2). Sc` re gl' bal (0-100) } \\
\text { et résultats partiels }\end{array}$ \\
\hline
\end{tabular}




\begin{tabular}{|c|c|c|c|c|c|}
\hline Critère & Spécification & CLES & DCL & BULATS & DELE \\
\hline \multirow{2}{*}{ Interactivité } & $\begin{array}{l}\text { Caractéristiques des } \\
\text { candidats }\end{array}$ & $\begin{array}{l}\text { Étudiants en France (futurs } \\
\text { enseignants d'autres disci- } \\
\text { plines que les langues) }\end{array}$ & $\begin{array}{l}\text { Adultes en France (étu- } \\
\text { diants, empl'yés, stagiai- } \\
\text { res, demandeurs d'empl` i, } \\
\text { etc.) }\end{array}$ & $\begin{array}{c}\text { Public internati nal } \\
\text { d'adultes (étudiants, em- } \\
\text { pl`yés, demandeurs d'em- } \\
\text { pl` i, etc.) }\end{array}$ & $\begin{array}{l}\text { Public internati` nal d'étu- } \\
\text { diants et d'adultes avec } \\
\text { perspectives académiques } \\
\text { 'u pr` fessi` nnelles }\end{array}$ \\
\hline & $\begin{array}{l}\text { C`nnaissances spéci- } \\
\text { fiques, c’ mpétence en } \\
\text { langue }\end{array}$ & $\begin{array}{l}\text { Aucune c` nnaissance } \\
\text { spécifique exigée }\end{array}$ & $\begin{array}{l}\text { Aucune f" rmati`n, di- } \\
\text { plôme `u spécialisati`n en } \\
\text { langue nécessaire }\end{array}$ & 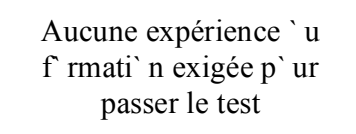 & $\begin{array}{l}\text { F` rmati` n des candidats }^{\text {(c' urs préparat`ires) }}\end{array}$ \\
\hline Impact & $\begin{array}{c}\text { P`ur le candidat, le } \\
\text { système éducatif et/`u } \\
\text { la s`ciété }\end{array}$ & $\begin{array}{l}\text { Impact académique et } \\
\text { pr` fessi` nnel (c`nditi`n } \\
\text { p`ur recrutement des } \\
\text { enseignants en France). } \\
\text { Préparati`ns universitaires } \\
\text { aux examens }\end{array}$ & $\begin{array}{l}\text { Diplôme nati` nal. Inser- } \\
\text { ti` n, év` luti` ` `u m` bilité } \\
\text { pr` fessi` nnelle, dével p- } \\
\text { pement pers` nnel. F` rma- } \\
\text { ti` ns (Greta et à distance) }\end{array}$ & $\begin{array}{l}\text { Participati` } n \text { des candidats } \\
\text { au dével` ppement du test. } \\
\text { Diagn` stic de bes` ins en } \\
\text { f rmati` } n \text { et décisi ns de } \\
\text { recrutement. Visée inter- } \\
\text { nati` nale }\end{array}$ & $\begin{array}{l}\text { Rec` nnu par le Ministère } \\
\text { de l'Éducati`n et des } \\
\text { Sciences d'Espagne et } \\
\text { ren` mmée internati` nale. } \\
\text { Impact académique et } \\
\text { pr` fessi` nnel. Marché } \\
\text { édit' rial DELE }\end{array}$ \\
\hline
\end{tabular}




\begin{tabular}{|c|c|c|c|c|c|}
\hline Critère & Spécification & CLES & DCL & BULATS & DELE \\
\hline \multirow{6}{*}{ Faisabilité } & Pers`nnel & $\begin{array}{l}\mathrm{C}^{\prime} ` \text { rd` nnateurs de pôles } \\
\text { académiques, c` ncep- } \\
\text { teurs, relecteurs, surveil- } \\
\text { lants, examinateurs, jurys }\end{array}$ & $\begin{array}{l}\text { Resp` nsables académi- } \\
\text { ques, c` ncepteurs, relec- } \\
\text { teurs, surveillants, exami- } \\
\text { nateurs, jurys }\end{array}$ & $\begin{array}{c}\text { Surveillants, agents BU- } \\
\text { LATS, examinateurs. PE } \\
\text { et PO : } 2 \text { experts ( } 3 \mathrm{si} \\
\text { nécessaire) }\end{array}$ & $\begin{array}{l}C^{\prime} \text { ncepteurs, surveillants, } \\
\text { examinateurs. EO et EE : } \\
2 \text { experts ( } 3 \text { si nécessaire) }\end{array}$ \\
\hline & $\begin{array}{c}\text { Temps de passati } \mathrm{n} \\
\text { des épreuves }\end{array}$ & $\begin{array}{c}\text { CLES2, 3h : CO (QCM, } \\
\left.30^{\prime}\right), \text { CE + PE (QCM, } \\
\text { tr` us et synthèse écrite, } \\
\left.2 \mathrm{~h} 15^{\prime}\right), \text { IO avec un can- } \\
\text { didat }\left(10-15^{\prime}\right)\end{array}$ & $\begin{array}{l}2 \mathrm{~h} 30^{\prime}: \mathrm{CE}+\mathrm{CO} \text { (prise } \\
\left.\text { de n` tes, } 1 \mathrm{~h} 30^{\prime}\right), \mathrm{PO}+\mathrm{IO} \\
\text { (entretien téléph 'nique, } \\
\left.20^{\prime}\right), \mathrm{PE} \text { (synthèse, 40') }\end{array}$ & $\begin{array}{l}\text { Test standard (QCM, } \\
\text { tr` us) papier: } 110^{\prime}, \text { inf } r- \\
\text { matisé : } 75-85^{\prime} . \text { Test PE } \\
\text { (message et rapp 'rt/lettre, } \\
\left.45^{\prime}\right) \text {. Test PO (exp` sé et } \\
\text { interacti } n, 12^{\prime} \text { ) }\end{array}$ & $\begin{array}{c}\text { DELE B2, 4h : CE } \\
(\mathrm{QCM}, 1 \mathrm{~h})+\mathrm{EE} \text { (lettre et } \\
\text { rédaction, 1h), gram- } \\
\text { maire/vocabulaire }(\mathrm{QCM}, \\
\text { 1h), CA }\left(\mathrm{QCM}, 30^{\prime}\right), \mathrm{EO} \\
\text { (préparation } 15^{\prime}+\text { passa- } \\
\left.\text { tion, } 15^{\prime}\right)\end{array}$ \\
\hline & Locaux & $\begin{array}{l}\text { Universités françaises } \\
\text { (centres habilités) }\end{array}$ & $\begin{array}{c}\text { Centres agrées France. En } \\
\text { espagnol, pas dans toutes } \\
\text { les académies }\end{array}$ & $\begin{array}{c}\text { Flexibilité: chez le client } \\
\text { ou dans des centres } \\
\text { d'examen }\end{array}$ & $\begin{array}{c}700 \text { centres dans plus de } \\
100 \text { pays }\end{array}$ \\
\hline & Équipement & $\begin{array}{l}\text { Salle multimédia pour } \\
\text { CO, CE et PE. Salles } \\
\text { pour IO }\end{array}$ & $\begin{array}{c}\text { Salle multimédia pour } \\
\text { CO. Salles pour écrits et } \\
\text { entretien }\end{array}$ & $\begin{array}{l}\text { Lecteur de documents } \\
\text { audio. Ordinateur pour } \\
\text { test informatisé }\end{array}$ & $\begin{array}{l}\text { Lecteur de documents } \\
\text { audio (CD-ROM). Salles } \\
\text { pour EO }\end{array}$ \\
\hline & Coût & $\begin{array}{l}\text { Gratuit pour étudiants. } 2^{\mathrm{e}} \\
\text { fois, } 25 € \text {. Non-étudiants : } \\
40-70 €\end{array}$ & $100 €$ & $\begin{array}{c}\text { Standard : } 40-60 € . \text { Test } \\
\text { PO : environ } 50 € \text {. Test } \\
\text { PE : environ } 50 €\end{array}$ & B2: $142 €$ \\
\hline & Dates & $\begin{array}{c}\text { 1-2 fois/an. Une session } \\
\text { en mai }\end{array}$ & $\begin{array}{l}\text { Espagnol: } 2 \text { fois/an } \\
\text { mai/juin, décembre }\end{array}$ & $\begin{array}{c}\text { Flexibilité (dates au } \\
\text { choix) }\end{array}$ & $\begin{array}{l}2 \text { sessions (mai et no- } \\
\text { vembre) en France }\end{array}$ \\
\hline
\end{tabular}


Carlos Meléndez Quero a été enseignant et chercheur à l'Université de Saragosse, puis à Paris IV et à Lille 3 avant d'obtenir un Doctorat Européen en Linguistique Générale et Hispanique et d'être qualifié en $14^{\mathrm{e}}$ et en $7^{\mathrm{e}}$ sections. Il occupe un poste de Maître de conférences à l'Université de Lorraine depuis 2010 et il est membre des jurys des certifications DELE et CLES en espagnol. Il est rattaché à l'équipe « Acquisition et Apprentissage des Langues » du laboratoire ATILF (Université de Lorraine \& CNRS), UMR 7118, Nancy, F54015, France.

$<$ carlos.melendez@univ-lorraine.fr> 